\title{
Role and mechanism of the Dectin-1-mediated Syk/NF-кB signaling pathway in Talaromyces marneffei infection
}

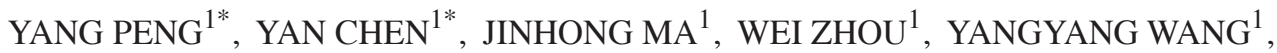 \\ YUYUE WANG ${ }^{1}$, HUI ZHENG ${ }^{2}$ and WEIFENG SHI ${ }^{1}$ \\ ${ }^{1}$ Department of Clinical Laboratory, The Third Affiliated Hospital of Soochow University, Changzhou, Jiangsu 213003; \\ ${ }^{2}$ Institutes of Biology and Medical Sciences, Soochow University, Suzhou, Jiangsu 215000, P.R. China
}

Received August 22, 2021; Accepted November 5, 2021

DOI: $10.3892 /$ etm.2021.11007

\begin{abstract}
Dendritic cell-associated C-type lectin-1 (Dectin-1), a C-type lectin receptor, serves a critical role in host antifungal immunity. However, the molecular mechanism and function of Dectin-1-mediated signaling in response to infection by the pathogenic fungus Talaromyces marneffei remains unclear. To understand the role of Dectin-1 signaling against T. marneffe $i$ infection, the phosphorylation of spleen tyrosine kinase (Syk), nuclear factor of $\kappa$ light polypeptide gene enhancer in B-cells inhibitor, $\alpha(\mathrm{I} \kappa \mathrm{B} \alpha)$ and $\mathrm{NF}-\kappa \mathrm{B}$ were analyzed using western blotting, and the secretion of cytokines was detected using ELISA. Upon sporular or hyphal heat-killed T. marneffei stimulation, Dectin-1 in THP-1 macrophages recognized and induced the activation of Syk, and in turn triggered phosphorylation of downstream molecules I $\kappa \mathrm{B} \alpha$ and $\mathrm{NF}-\kappa \mathrm{B}$, thus increasing the secretion of TNF- $\alpha$ and IL-8. Conversely, knockdown of Dectin-1 in THP-1 macrophages downregulated the phosphorylation of $\mathrm{Syk}, \mathrm{I} \kappa \mathrm{B} \alpha$ and $\mathrm{NF}-\kappa \mathrm{B}$ molecules, and significantly decreased the production of TNF- $\alpha$ and IL- 8 . These results indicated that Dectin-1 may have a crucial role in inducing the inflammatory response via increasing levels of TNF- $\alpha$ and IL-8 induced by T. marneffei, whereas NF- $\kappa \mathrm{B}$ may be the key downstream molecule involved in the response to T. marneffei infection. Subsequently, THP-1 macrophages could orchestrate the innate immune system by releasing the cytokines TNF- $\alpha$ and IL-8. Therefore, it was hypothesized that regulation of the Dectin-1 signaling pathway may effectively interfere with the defense ability of the host against T. marneffei infection.
\end{abstract}

Correspondence to: Professor Weifeng Shi, Department of Clinical Laboratory, The Third Affiliated Hospital of Soochow University, 185 Juqian Road, Changzhou, Jiangsu 213003, P.R. China

E-mail: shiweifeng67@163.com

${ }^{*}$ Contributed equally

Key words: Talaromyces marneffei, macrophages, dendritic cell-associated C-type lectin-1, cytokines

\section{Introduction}

Talaromycosis is a severe deep mycosis caused by Talaromyces marneffei, which was first isolated from a bamboo rat in Vietnam in 1956 by Capponi et al (1). T. marneffei is a thermally dimorphic endemic fungus. When cultured on SDA medium at $25^{\circ} \mathrm{C}$, the colony appears velvety gray-green, and diffuses a red pigment into the culture medium; however, at $37^{\circ} \mathrm{C}$, the spores convert to a pathogenic yeast phase, and no diffusing pigment is produced (2). T. marneffei infections are usually initiated by the inhalation of dormant spores, which are produced outside of the host during the differentiation of the hyphal growth form. In the lungs, host innate immune cells recognize these propagules $(3,4)$. Notably, $T$. marneffei causes disseminated infection in immunocompromised patients, particularly in individuals of Southeast Asian descent and southern China (5). The fungus is one of the leading causes of death among immunocompromised patients. For infected patients, early clinical diagnosis is difficult, and the effectiveness of antifungal therapy is often limited, resulting in high rates of mortality and morbidity $(6,7)$. To date, the underlying immunological mechanisms involved in the recognition and control of T. marneffei are unclear.

Innate immunity represents the first line of defense for hosts against microbes. Upon invasion, pathogens are suppressed by the host innate immune system through the recognition of pathogen-associated molecular patterns (PAMPs) by pattern recognition receptors (PRRs). It is well established that NOD-like receptors (NLRs), Toll-like receptors (TLRs), C-type lectin receptors (CLRs) and retinoic acid-inducible gene I-like receptors are the best characterized PRRs for sensing different types of PAMPs $(8,9)$. Among these, CLRs are one of the most important PRRs that detect fungi in the innate immune system (10). Specifically, the CLRs consists of dendritic cell (DC)-associated C-type lectin-1 (Dectin-1, CLEC7A), Dectin-2 (CLEC4N), mannose receptor (CD206), macrophage-inducible C-type lectin (CLEC4E), macrophage C-type lectin (CLEC4D), melanin-sensing C-type lectin (CLEC1A) and DC-specific intercellular adhesion molecule-3-grabbing nonintegrin (CD209) (11). As previously reported, CLRs are primarily expressed on myeloid cells, including macrophages, DCs and neutrophils (12). Of interest is Dectin-1, which is a key protein involved in the CLR-mediated antifungal signaling 
pathway, recruiting additional proteins to form a multiprotein complex capable of activating the $\mathrm{NF}-\kappa \mathrm{B}$ inflammatory pathway. Dectin-1 is a type II transmembrane protein, which recognizes $\beta$-1,3-glucans in the cell wall of various pathogenic fungi (13). It may stimulate several cellular responses via the spleen tyrosine kinase (Syk)/CARD9 signaling pathway, such as phagocytosis, the production of cytokines and the respiratory burst (14). Furthermore, it is a major recognition receptor for various types of fungi, including species of Aspergillus, Candida, Histoplasma and Cryptococcus, among others (15).

Despite Dectin-1 playing an important role in regulating host immunity and fungal infection, the activation of Dectin-1 induced by $T$. marneffei infection remains to be elucidated. The aim of the current study was to explore role and mechanism of Dectin-1-mediated signaling pathway in T. marneffei infection using THP-1 macrophages.

\section{Materials and methods}

Reagents and antibodies. RPMI-1640 medium, FBS, penicillin-streptomycin solution and $\beta$-mercaptoethanol were purchased from Gibco (Thermo Fisher Scientific, Inc.). TRIzol ${ }^{\circledR}$ reagent was obtained from Invitrogen (Thermo Fisher Scientific, Inc.). PMA and puromycin were purchased from Sigma-Aldrich (Merck KGaA). One Step TB Green ${ }^{\mathrm{TM}}$ Prime Script $^{\mathrm{TM}}$ RT-PCR kit II was purchased from Takara Bio, Inc. RIPA lysis buffer was purchased from Beijing Solarbio Science \& Technology Co., Ltd. BSA, BCA assay and blocking buffer were purchased from Beyotime Institute of Biotechnology. ECL was purchased from Bio-Rad Laboratories, Inc. Dectin-1 short hairpin (sh) RNA lentiviral particles and scramble shRNA lentiviral particles encoding a GFP sequence were constructed by Shanghai GeneChem Co., Ltd. Antibodies against Dectin-1 (cat. no. 60128), NF- $\kappa$ B p65 (cat. no. 8242), phosphorylated

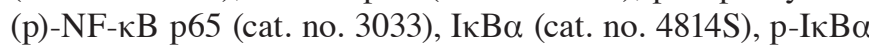
(cat. no. 9246), Syk (cat. no. 2712), p-Syk (cat. no. 2710) and anti-rabbit $\operatorname{IgG}(\mathrm{H}+\mathrm{L})$, Alexa Fluor ${ }^{\circledR}$ 555-conjugated anti-rabbit IgG (cat. no. 4413) were purchased from Cell Signaling Technology, Inc.; horseradish peroxidase (HRP)-conjugated goat anti-rabbit IgG secondary antibody (cat. no. L3012 was purchased from Signalway Antibody LLC; HRP-conjugated goat anti-mouse IgG secondary antibody (cat. no. ab6789) was purchased from Abcam; and anti- $\beta$-actin (cat. no. 66009-1-Ig) was purchased from ProteinTech Group, Inc.

Cell culture and maintenance. The human monocyte cell line THP-1 (The Cell Bank of Type Culture Collection of The Chinese Academy of Sciences) was cultured in RPMI-1640 medium supplemented with $10 \%$ inactivated FBS, $0.05 \mathrm{mM}$ $\beta$-mercaptoethanol and $100 \mathrm{U} / \mathrm{ml}$ penicillin-streptomycin solution in a humidified atmosphere containing $5 \% \mathrm{CO}_{2}$ at $37^{\circ} \mathrm{C}$. THP- 1 cells could be differentiated into macrophages by treatment with $100 \mathrm{ng} / \mathrm{ml} \mathrm{PMA}$ at $37^{\circ} \mathrm{C}$ for $48 \mathrm{~h}$. The cells were then cultured in medium without PMA.

Fungal culture. T. marneffei [CMCC(F)B33r; The Chinese Academy of Medical Sciences and Peking Union Medical College] strains were cultivated on potato dextrose agar slants and incubated for 3-7 days at 25 or $37^{\circ} \mathrm{C}$. Fungal spores or hyphae were harvested by washing the plates with sterile
PBS with $0.01 \%$ Tween-80 (PBST) solution. The hyphae were ground to obtain $20-40 \mu \mathrm{m}$ fragments. The suspension was then gently filtered through a $40-\mu \mathrm{m}$ nylon pore mesh cell strainer. Subsequently, the spores or hyphae were thoroughly washed, centrifuged for $5 \mathrm{~min}$ at 2,000 x g at room temperature, resuspended in sterile PBST, and adjusted to a concentration of $1 \times 10^{8} \mathrm{CFU} / \mathrm{ml}$ with RPMI-1640 medium. As required, spores and hyphae were killed by heating at $65^{\circ} \mathrm{C}$ in water for $2 \mathrm{~h}$ (16). Finally, the suspension was stored at $4^{\circ} \mathrm{C}$ for use within $48 \mathrm{~h}$. THP-1 macrophages were incubated with heat-killed T. marneffei spores or hyphae (25:1, fungi to cell) cultured at $37^{\circ} \mathrm{C}$ in a $5 \% \mathrm{CO}_{2}$ atmosphere for $0,1,2,4,8$ and 12 h. THP-1 macrophages with Dectin-1 expression silencing using a shRNA-Dectin-1 interference and transfected with an ineffective interfering sequence were incubated with heat-killed T. marneffei spores (25:1, fungi to cell) and cultured at $37^{\circ} \mathrm{C}$ in a $5 \% \mathrm{CO}_{2}$ atmosphere for the indicated time periods.

PCR array. This study used a customized PCR array from CT Bioscience to analyze the expression of key genes that participated in T. marneffei-induced immune response. In brief, THP-1 macrophages were harvested after treatment with or without T. marneffei spores or hyphae for $8 \mathrm{~h}$. A total of 88 potential genes that may be involved in T. marneffei-induced cellular antifungal immune responses were selected as the target mRNAs. After RNA isolation, $1 \mu \mathrm{g}$ total RNA was used for reverse transcription using an RT kit (cat. no. CTB101; CT Bioscience) in a $20 \mu \mathrm{l}$ volume, according to the manufacturer's protocols. The PCR array employed SYBR Green I-based quantitative PCR (qPCR) (cat. no. CTB103; CT Bioscience) to quantify gene expression level. qPCR was performed in a Roche LightCycler s480-II instrument (Roche Diagnostics) under the following thermocycling conditions: $10 \mathrm{~min}$ at $93^{\circ} \mathrm{C}$, followed by 45 cycles of $10 \mathrm{sec}$ at $93^{\circ} \mathrm{C}$ and $30 \mathrm{sec}$ at $60^{\circ} \mathrm{C}$.Gene specific primers were pre-deposited into wells of a 96-well PCR plate in the array. GAPDH, $\beta$-2-microglobulin (B2M), ACTB, hypoxanthine-guanine phosphoribosyltransferase (HPRT1) and ornithine decarboxylase antizyme 1 (OAZ1) were used as housekeeping genes for normalization. The primers used were as follows: C-ros oncogene 1 receptor tyrosine kinase (ROS) forward, 5'-GCAAATA ATCTAGGGTTTGGTGA-3' and reverse, 5'-TCAGTGGGAT TGTAACAACCAG-3'; NLR family pyrin domain-containing (NLRP)11 forward, 5'-TGTTCAGCGCATCTTTCAA-3' and reverse, 5'-CTCCAGTAGACAAGGCTCTTCA-3'; Dectin-1 forward, 5'-AGCCTACCTGTAGGTCGACAA-3' and reverse, 5'-CTGAGGTCAAGATAAATGCAGAAA-3'; Dectin-3 forward, 5'-CCAGCTGATACCTTCGGTTA-3' and reverse, 5'-TGCCTCTCTTACAGCGTGAA-3'; NLRP3 forward, 5'-TGAAGTGCTGAAACAGCAGAG-3' and reverse, 5'-AAA GACGACGGTCAGCTCAG-3'; TNF- $\alpha$ forward, 5'-GCCCG ACTATCTCGACTTTG-3' and reverse, 5'-ATGTTCGTCCT CCTCACAGG-3'; IL-8 forward, 5'-AAGACATACTCCAAAC CTTTCCAC-3' and reverse, 5'-AATTTCTGTGTTGGCGC AGT-3'; B2M forward, 5'-TGTCTTTCAGCAAGGACTGG-3' and reverse, 5'-AACTATCTTGGGCTGTGACAAA-3'; ACTB forward, 5'-AGTCCGCCTAGAAGCATTTG-3' and reverse, 5'-CTGTCCACCTTCCAGCAGAT-3'; HPRT1 forward, 5'-ACGTCTTGCTCGAGATGTGA-3' and reverse, 5'-AATCC AGCAGGTCAGCAAAG-3'; OAZ1 forward, 5'-GGAACCGT 
AGACTCGCTCAT-3' and reverse, 5'-TGAGCGTTTATTTGC ACGAT-3'; and GAPDH forward, 5'-GGGAGCCAAAAGGGT CATCA-3' and reverse, 5'-TGGTTCACACCCATGACGAA-3'.

Lentiviral transfection of THP-1 cells. The design of the Dectin-1 shRNA (interference sequence, 5'-CAATTACAC TTCGACTCTCAA-3'), a scrambled shRNA (5'-TTCTCCGA ACGTGTCACGT-3') and the packaging of the lentivirus particles were performed by Shanghai GeneChem Co., Ltd. A total of $4 \times 10^{4}$ THP- 1 cells were cultured in supplemented RPMI medium in 96-well plates for $24 \mathrm{~h}$. Subsequently, $4 \mu \mathrm{l}$ HitransG P (25X; Shanghai GeneChem Co., Ltd.) enhanced infection solution was added prior to cell transduction with lentiviral particles at a multiplicity of infection (MOI) of 50 (virus number/cell number $=50$ ). Cells were incubated for $12 \mathrm{~h}$, and then the culture medium was replaced with fresh medium. GFP expression was observed by fluorescence microscopy $72 \mathrm{~h}$ after transduction. After $72 \mathrm{~h}$ of infection, fresh medium containing $2 \mu \mathrm{g} / \mathrm{ml}$ puromycin was added for $72 \mathrm{~h}$ to select positively stably transduced cells. Stably transduced cells were maintained in $1 \mu \mathrm{g} / \mathrm{ml}$ puromycin. The third passage of stable clones was collected at 9 days after transduction for reverse transcription (RT)-qPCR analysis.

$R T-q P C R$. THP-1 macrophages were incubated with heat-killed T. marneffei spores or hyphae (25:1, fungi to cell) cultured at $37^{\circ} \mathrm{C}$ in $5 \% \mathrm{CO}_{2}$ for $0,1,2,4,8$ and $12 \mathrm{~h}$. Following these treatments, cells were harvested, and total RNA was extracted using TRIzol ${ }^{\circledR}$ reagent. RT-qPCR was performed using a One Step TB Green ${ }^{\mathrm{TM}}$ Prime Script ${ }^{\mathrm{TM}}$ RT-PCR kit II on an Applied Biosystems 7500 Real-Time PCR system (Applied Biosystems; Thermo Fisher Scientific, Inc.). GAPDH was used as the internal control. The thermocycling conditions were as follows: Reverse transcription at $42^{\circ} \mathrm{C}$ for $5 \mathrm{~min}$; pre-denaturation for $10 \mathrm{sec}$ at $95^{\circ} \mathrm{C}$; followed by 40 cycles of $5 \mathrm{sec}$ at $94^{\circ} \mathrm{C}$ and $34 \mathrm{sec}$ at $60^{\circ} \mathrm{C}$. The specific primers used were Dectin-1 forward, 5'-CAACTGGGCTCTAATCTCC-3' and reverse, 5'-GCACACTACACAGTTGGTC-3'; and GAPDH forward, 5'-GACCTGACCTGCCGTCTA-3' and reverse 5'-AGGAGTGGGTGTCGCTGT-3'. Finally, the Cq values for each reaction were collected and the changes in the expression of the target gene were normalized to GAPDH. Relative mRNA expression levels were calculated by fold changes using the $2^{-\Delta \Delta C q}$ formula, where $\Delta \mathrm{Cq}$ is the difference between the target gene and GAPDH, and $\Delta \Delta \mathrm{Cq}$ for the sample $=\Delta \mathrm{Cq}$ of treated condition $-\Delta \mathrm{Cq}$ of control condition (17).

Western blotting. THP-1 macrophages were incubated with heat-killed T. marneffei spores or hyphae (25:1, fungi to cell) cultured at $37^{\circ} \mathrm{C}$ in $5 \% \mathrm{CO}_{2}$ for $0,1,2,4,8$ and $12 \mathrm{~h}$. After treatment, cells were lysed in RIPA lysis buffer containing protease inhibitor cocktail and the phosphatase inhibitor PhosSTOP (cat. no. 78440; Thermo Fisher Scientific, Inc.). The protein concentration was determined using a BCA assay. Total protein $(20 \mu \mathrm{g} /$ lane) was separated by $10 \%$ SDS-PAGE and transferred to a PVDF membrane (MilliporeSigma). After blocking with $5 \%$ skimmed milk for $1.5 \mathrm{~h}$ at room temperature, the membranes were incubated with the following primary antibodies at $4^{\circ} \mathrm{C}$ overnight: Anti-Dectin-1 (1:1,000), anti-NF- $\mathrm{KB}$

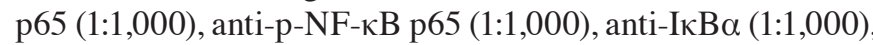

anti-p-IкB $\alpha(1: 1,000)$, anti-Syk $(1: 1,000)$, anti-p-Syk $(1: 1,000)$ and anti- $\beta$-actin $(1: 5,000)$. The membranes were then incubated with the appropriate HRP-conjugated secondary antibodies $(1: 5,000)$ at $37^{\circ} \mathrm{C}$ for $1 \mathrm{~h}$. The immunoreactive bands were visualized with ECL reagents.

Immunofluorescence assay for detecting the nuclear translocation of $N F-\kappa B$. Cells were cultured on glass dishes. THP-1 macrophages were incubated with heat-killed T. marneffei spores for $4 \mathrm{~h}$, and THP-1 macrophages with Dectin-1 shRNA or scrambled shRNA were incubated with heat-killed T. marneffei spores for $4 \mathrm{~h}$. Stimulated and unstimulated THP-1 macrophages were washed three times with PBS at various time points, fixed with $4 \%$ paraformaldehyde at $37^{\circ} \mathrm{C}$ for $30 \mathrm{~min}$, and permeabilized with $0.2 \%$ Triton $\mathrm{X}-100$ for $15 \mathrm{~min}$, and then washed with PBS. After blocking with 5\% BSA in PBS for $30 \mathrm{~min}$ at $37^{\circ} \mathrm{C}$, the cells were incubated with rabbit anti-NF- $\mathrm{kB}-\mathrm{p} 65$ antibodies at $4^{\circ} \mathrm{C}$ overnight. The cells were washed with PBST, and then incubated with Alexa 555-conjugated anti-rabbit $\operatorname{IgG}$ at $37^{\circ} \mathrm{C}$ for $1 \mathrm{~h}$. Finally, using a drop of ProLong ${ }^{\mathrm{TM}}$ Diamond antifade mountant medium with DAPI (cat. no. P36962; Invitrogen; Thermo Fisher Scientific, Inc.) nuclei were stained for $10 \mathrm{~min}$ at room temperature and sealed. The slides were carefully observed under a confocal microscope.

Cytokine quantification using ELISA. After stimulation of THP-1 macrophages $\left(5 \times 10^{5}\right.$ cells $\left./ \mathrm{ml}\right)$ with heat-killed T. marneffei spores for 8 or $18 \mathrm{~h}$, specific commercial ELISA kits (R\&D Systems, Inc.) were used to measure the quantity of TNF- $\alpha$ (cat. no. DTA00D) and IL-8 (cat. no. D8000C) in the cell-free culture supernatants. The experimental procedure was performed according to the manufacturer's protocols. All experiments were performed in triplicate. Data are presented as the mean \pm standard deviation.

Statistical analysis. Experiments were conducted at least three times. Data are presented as the mean \pm standard deviation. Differences among the groups were evaluated using one-way ANOVA followed by Tukey's post hoc test. Intragroup (time) and intergroup (fluences) comparisons of the Cytokine quantification were analyzed by two-way ANOVA followed by Tukey's post hoc test using SPSS version 22.0 (IBM Corp.). $\mathrm{P}<0.05$ was considered to indicate a statistically significant difference.

\section{Results}

Dectin-1 expression is increased following T. marneffei infection in vitro. To identify the potential genes that may be involved in T. marneffei infection, an in vitro model of THP-1 macrophages infected with heat-killed T. marneffei spores and hyphae was established, and a PCR-array was used to screen differential gene expression in the THP-1 macrophages together with or without $T$. marneffei spores or hyphae. It was revealed that the expression levels of ROS, Dectin-3 and NLRP11 were downregulated, whereas the levels of Dectin-1, NLRP3, TNF- $\alpha$ and IL-8 were upregulated compared with untreated THP-1 macrophages (Fig. 1A). Since Dectin-1 had previously been suggested to serve important roles in 
A

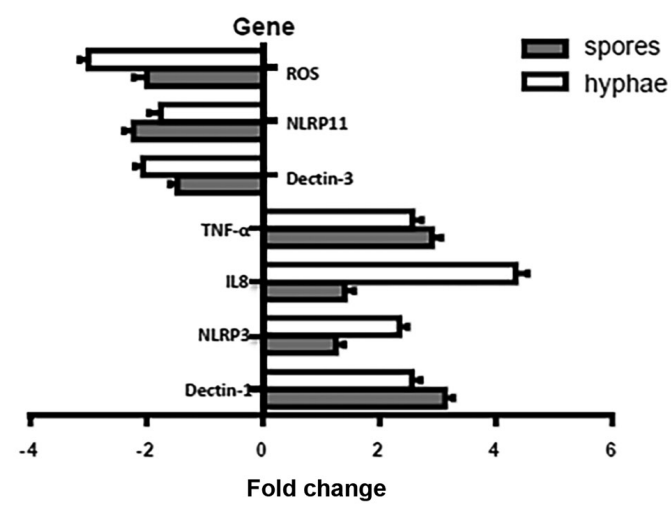

B

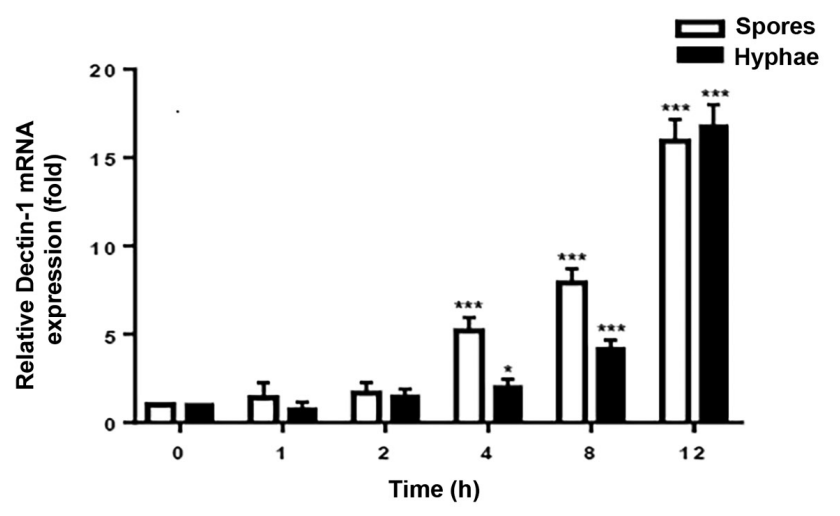

C

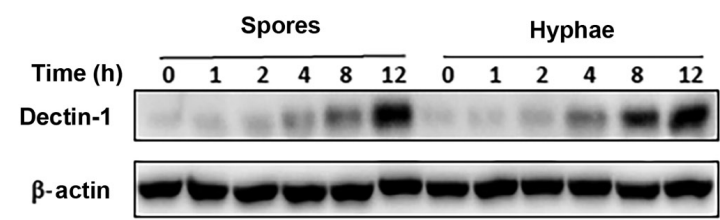

Figure 1. Dectin-1 expression is increased following Talaromyces marneffei infection in vitro. (A) PCR-array analysis of the expression levels of different genes in THP-1 macrophages infected with T. marneffei for 8 h. (B) Dectin-1 expression in THP-1 macrophages infected with T. marneffei spores or hyphae were analyzed by quantitative PCR at different time points. (C) Western blot analysis of Dectin-1 expression in T. marneffei-infected THP-1 macrophages at different time points post-infection. mRNA expression are relative to GAPDH expression and are presented as the mean \pm standard deviation of three repeats. $\beta$-actin was used as the western blotting loading control. ${ }^{*} \mathrm{P}<0.05$ and ${ }^{* * *} \mathrm{P}<0.001$ vs. $0 \mathrm{~h}$. NLRP, NLR family pyrin domain-containing; Dectin-1, dendritic cell-associated C-type lectin-1; ROS, C-ros oncogene 1 receptor tyrosine kinase.

the antifungal response, a focus was placed on the study of Dectin-1 and its regulatory roles in the antifungal immune response to $T$. marneffei infection. To investigate whether Dectin-1 expression was affected by T. marneffei infection in vitro, THP-1 macrophages were infected with heat-killed T. marneffei spores or hyphae for various durations. The expression levels of Dectin-1 in THP-1 macrophages were examined using RT-qPCR and western blotting. The relative mRNA expression levels of Dectin-1 in THP-1 macrophages were significantly increased in response to T. marneffei infection. Compared with in the uninfected control cells, Dectin-1 mRNA expression levels were significantly increased 4,8 and $12 \mathrm{~h}$ after infection with heat-killed T. marneffei spores or hyphae (Fig. 1B). Western blotting results revealed that Dectin-1 protein expression levels were elevated 4, 8 and $12 \mathrm{~h}$ following infection with heat-killed T. marneffei spores and hyphae (Fig. 1C). Western blotting and RT-qPCR results confirmed that the expression levels of Dectin-1 were gradually increased in the cells with the prolongation of stimulation time. These results suggested that $T$. marneffei infection may promote the expression levels of Dectin-1 in vitro.

T. marneffei triggers the activation of $S y k / N F-\kappa B$ signaling pathways. To study the Dectin-1 expression patterns in the antifungal immune response, THP-1 macrophages were exposed to heat-killed T. marneffei spores or hyphae for 1, 2, 4, 8 or $12 \mathrm{~h}$. Western blotting was used to analyze Dectin-1 protein expression levels in THP-1 macrophages. It was observed that Dectin-1 protein expression levels were upregulated in the THP-1 macrophages stimulated with spores and hyphae compared with in the control cells (Fig. 1C). The phosphorylation levels of Syk, p65 and $\mathrm{I} \kappa \mathrm{B} \alpha$ were detected using western blotting, and were increased in a time-dependent manner; however, I $\mathrm{B} \alpha$ phosphorylation began to decrease slightly at $4 \mathrm{~h}$ poststimulation (Fig. 2A). After Dectin-1 expression was knocked down, the phosphorylation levels of Syk, p65 and I $\kappa \mathrm{B} \alpha$ were inhibited (Fig. 2B). These results indicated that activation of Syk/NF- $\mathrm{B}$ may be involved in the T. marneffei-induced inflammatory response.

T. marneffei induces the translocation of NF- $\kappa B$ in THP-1 macrophages. To further confirm the effect of T. marneffe $i$ on NF- $\kappa \mathrm{B}$ activation in THP-1 macrophages, immunofluorescence analysis was used to assess the cellular localization of $\mathrm{NF}-\kappa \mathrm{B}$ in THP-1 macrophages. The stimulated or unstimulated THP-1 macrophages, fixed $4 \mathrm{~h}$ after stimulation, were stained with diluted rabbit anti-NF- $\mathrm{B}-\mathrm{p} 65$ antibodies. As revealed in Fig. 3, T. marneffei induced the translocation of $\mathrm{NF}-\kappa \mathrm{B}$ in THP-1 macrophages. As shown in Fig. 3A, NF- $\mathrm{B}-$ p65 was primarily localized in the cytoplasm of normal untreated cells, whereas it was predominately located in the nuclei of the T. marneffei-stimulated cells. Following knockdown of Dectin-1 expression, the nuclear translocation of $\mathrm{p} 65$ protein was inhibited (Fig. 3B). Compared with in the SCR + spores group, the fluorescence intensity of nuclear p65 in THP-1 macrophages was reduced in the shDectin-1 + spores group. These results demonstrated that T. marneffei elicited inflammatory activity in THP-1 macrophages by modulating subcellular localization of the transcriptional factor $\mathrm{NF}-\kappa \mathrm{B}$.

Knockdown of Dectin-1 inhibits cytokine release from macrophages. Since the expression of inflammatory cytokines is regulated by $\mathrm{NF}-\kappa \mathrm{B}$, the levels of TNF- $\alpha$ and IL- 8 were finally detected using an ELISA. The interaction between T. marneffei and THP-1 macrophages resulted in increased secretion of TNF- $\alpha$ and IL-8 (Fig. 4A and B). THP-1 macrophages were transfected with Dectin-1 shRNA or scrambled shRNA. Two-way ANOVA analysis demonstrated that knockdown of Dectin-1 had statistical significance at the IL-8 level and TNF- $\alpha$ level (data not shown). The Dectin-1 shRNA group exhibited lower IL- 8 and TNF- $\alpha$ levels than in the SCR group. Knockdown of Dectin-1 in THP-1 macrophages decreased the 
A

\begin{tabular}{|c|c|c|c|c|c|c|c|c|c|c|c|c|}
\hline \multirow[b]{2}{*}{ Time $(h)$} & \multicolumn{6}{|c|}{ Spores } & \multicolumn{6}{|c|}{ Hyphae } \\
\hline & 0 & 1 & 2 & 4 & 8 & 12 & 0 & 1 & 2 & 4 & 8 & 12 \\
\hline p-Syk & $=$ & $m$ & 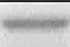 & $=$ & $=$ & $=$ & $=2$ & 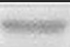 & $m$ & 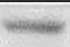 & $=$ & $=$ \\
\hline Syk & & & & & & & & - & & & $=$ & $\longrightarrow$ \\
\hline p-p65 & - & - & & & & & - & 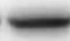 & $=$ & 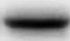 & $=$ & $\longrightarrow$ \\
\hline p65 & - & 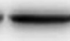 & - & - & - & $\longrightarrow$ & - & 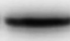 & 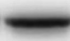 & - & 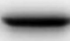 & 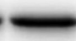 \\
\hline $\mathrm{p}-\mathrm{|kB} \alpha$ & & $=$ & L & 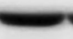 & 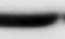 & $=$ & 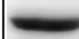 & $=$ & $=$ & 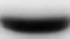 & 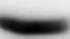 & $=$ \\
\hline IK $B \alpha$ & - & 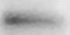 & teos & $m$ & $m$ & 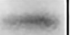 & $m$ & $\mathrm{~mm}$ & $m$ & $m$ & $m$ & 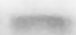 \\
\hline$\beta$-actin & & & & & & & & & & & & \\
\hline
\end{tabular}

B

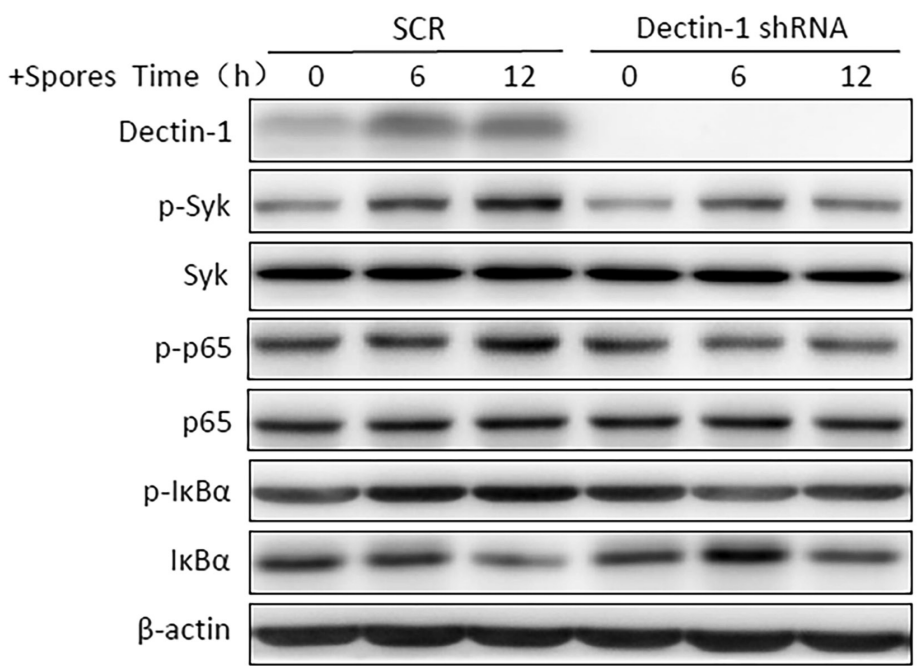

Figure 2. Talaromyces marneffei triggers the activation of the Syk/NF- $\mathrm{kB}$ signaling pathway. (A) THP-1 macrophages were incubated with heat-killed T. marneffei spores or hyphae for the indicated time periods. (B) THP-1 macrophages were transfected with Dectin-1 shRNA or SCR shRNA. Cells were then incubated with T. marneffei spores for the indicated time. Syk, p65 and IкB $\alpha$ activation were determined using immunoblotting with anti-p-Syk, anti-p-p65

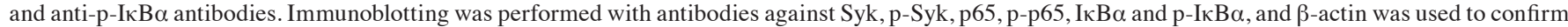
equal protein loading. p, phosphorylated; Syk, spleen tyrosine kinase; I $\mathrm{B} \alpha$, nuclear factor of $\kappa$ light polypeptide gene enhancer in B-cells inhibitor, $\alpha$; SCR, scrambled; shRNA, short hairpin RNA; Dectin-1, dendritic cell-associated C-type lectin-1.

production of TNF- $\alpha$ and IL-8. Dynamic changes of IL-8 and TNF- $\alpha$ levels are presented in Fig. $4 \mathrm{C}$ and D. These results demonstrated that Dectin-1 may be involved in the release of cytokines from macrophages following fungal stimulation, and supported the hypothesis that Dectin-1 may be pivotal in the recognition of T. marneffe $i$ and subsequent macrophage activation.

Proposed mechanism of Dectin-1 involvement in the recognition of T. marneffei on human macrophages and contribution to their immunomodulatory capacity. The results of the present study indicated that $T$. marneffei bind and activate the Dectin-1 receptor, leading to activation of the NF- $\mathrm{\kappa B}$ signaling pathway, including activation of IKK complexes, release of $\mathrm{p} 50 / \mathrm{p} 65$ complexes from the inhibitor complex with IкB $\alpha$ and translocation of the phosphorylated p50/p65 heterocomplex to the nucleus, where the transcription of proinflammatory genes, including those for cytokines, such as TNF- $\alpha$ and IL-8, was promoted (Fig. 5).

\section{Discussion}

Due to the lack of effective antifungal agents, talaromycosis is well known as a severe disease that can cause disseminated infection, and treatment of this disease remains a challenge (18). Nakamura et al (19) demonstrated that Dectin-1 was essential in sensing T. marneffei for the activation of bone marrow-derived DCs. However, the interaction between macrophages and T. marneffei remains largely unknown. Innate immunity acting as the front-line defense plays an essential role in resisting fungal infections. Dectin-1 is a member of the C-type lectin family and functions as an innate PRR involved in antifungal immunity. Although the role of Dectin-1 in mediating talaromycosis remains poorly understood, several studies have indicated its role in antifungal immunity. Gantner et al (20) reported that, via $\beta$-glucan-containing particles, Dectin-1 expression enhanced TLR-mediated activation of NF- $\kappa$ B. In addition, in macrophages and DCs, Dectin-1 and TLRs were revealed to be 
A

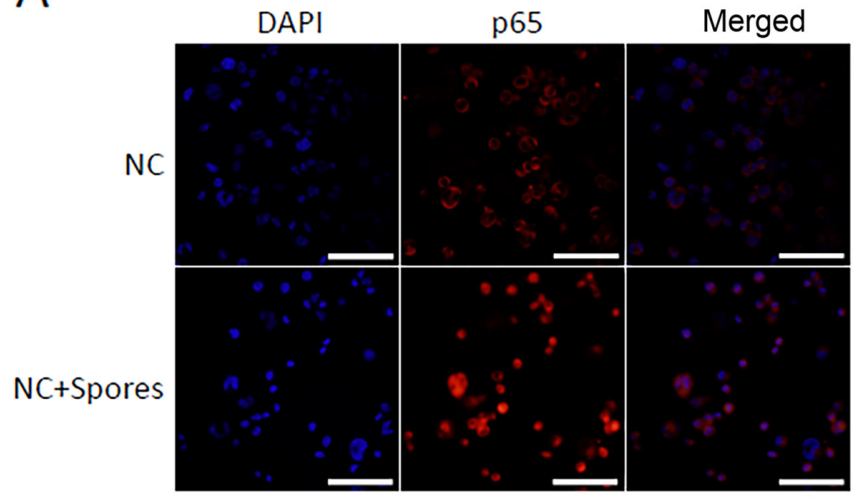

B

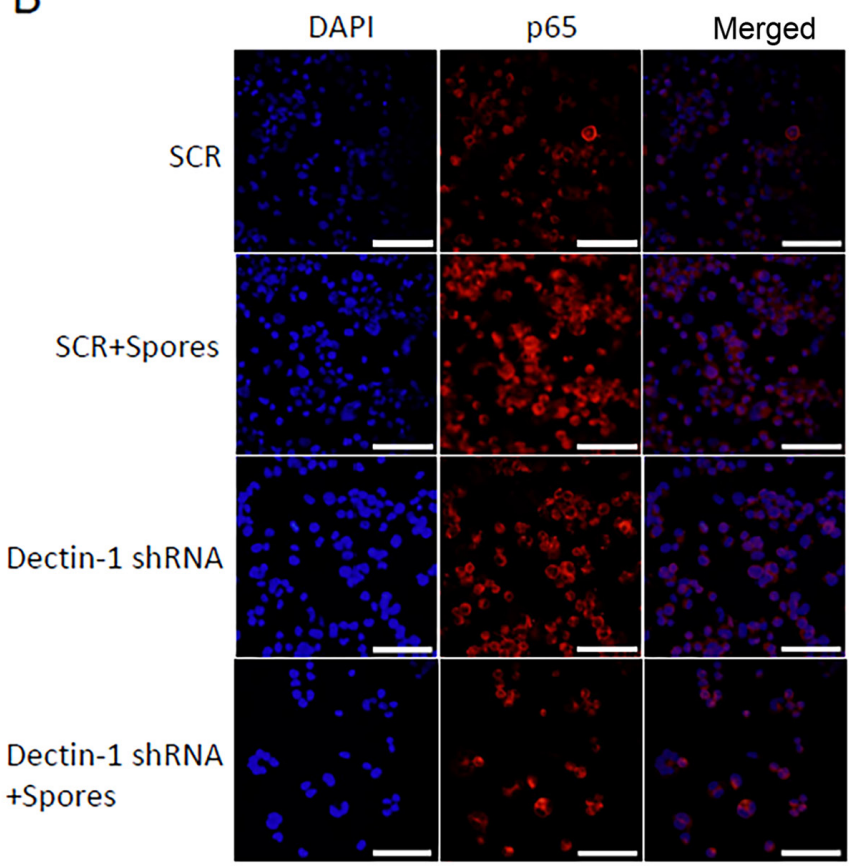

Figure 3. Talaromyces marneffei induces the translocation of $\mathrm{NF}-\kappa \mathrm{B}$ in THP-1 macrophages. (A) Confocal microscopic images demonstrated the translocation of NF- $\kappa \mathrm{B}$ in THP-1 macrophages following treatment with T. marneffei for $4 \mathrm{~h}$. NF- $\mathrm{B}$ p 65 translocation was analyzed by staining with an antibody against NF-кB-p65 (red), and nuclei were stained with DAPI (blue). The merged images revealed that $T$. marneffei could induce the translocation of NF- $\mathrm{B}$ in THP-1 macrophages. (B) THP-1 macrophages were transfected with Dectin-1 shRNA or SCR shRNA control. Transfected cells were then incubated with T. marneffei spores for $4 \mathrm{~h}$. Scale bar, $100 \mu \mathrm{m}$. SCR, scrambled; shRNA, short hairpin RNA; NC, normal control; Dectin-1, dendritic cell-associated C-type lectin-1.

synergistic in mediating the production of cytokines, such as IL-12 and TNF. Sun et al (21) demonstrated that Dectin-1 may serve an important role in Aspergillus-induced innate immune responses in human bronchial epithelial cells. In addition, Cohen-Kedar et al (22) presented evidence for $\beta$-glucan- and fungal-induced activation of human intestinal epithelial cells and the potential underlying mechanism, which involved the Dectin-1/Syk pathway. These findings indicated that Dectin-1 may be a critical component of the antifungal immune response in macrophages. Therefore, in the present study, the role and mechanism of Dectin-1 in T. marneffei infection of macrophages was investigated.
To investigate whether Dectin-1 recognized the spores and hyphae of T. marneffei, PMA-induced THP-1 macrophages stimulated with T. marneffei spores or hyphae were used, and the expression levels of Dectin-1 mRNA and protein were determined. In the present study, it was revealed that THP-1 macrophages interacted with distinct T. marneffei morphotypes, and increased expression levels of Dectin-1 were observed in macrophages in response to T. marneffe $i$ infection. These findings suggested that Dectin-1 was involved in the recognition of T. marneffei by macrophages.

$\mathrm{NF}-\kappa \mathrm{B}$ is an important transcriptional regulator, which controls the expression of various pro-inflammatory mediators. The NF- $\kappa \mathrm{B}$ family of transcription factors consists of five members, p50, p52,p65 (RelA), c-Rel and RelB. The underlying mechanism of NF- $\kappa \mathrm{B}$ signaling consists of a series of positive and negative regulatory elements. Firstly, inducing stimuli initiate IKK activation leading to phosphorylation, ubiquitination and degradation of $\mathrm{I} \kappa \mathrm{B}$ proteins. $\mathrm{I} \kappa \mathrm{B}$ is an inhibitory protein that acts to prevent $\mathrm{NF}-\kappa \mathrm{B}$ migration into the nucleus, and the degradation of $\mathrm{I} \kappa \mathrm{B}$ leads to the release of $\mathrm{NF}-\kappa \mathrm{B}$ p $65 / \mathrm{p} 50$ dimers into the nucleus $(23,24)$. Zhu et al $(25)$ demonstrated that stimulation of RAW264.7 cells with Candida albicans hyphae triggered Syk phosphorylation and $\mathrm{I} \kappa \mathrm{B} \alpha$ degradation. Sun et al (26) identified that Aspergillus fumigatus infection induced I $\kappa \mathrm{B} \alpha$ phosphorylation and NF- $\kappa \mathrm{B}$-mediated activation of THP-1 macrophages. Furthermore, Rogers et al (27) revealed the classical Syk-dependent $N F-\kappa B$ pathway, and showed that following zymosan binding to Dectin-1, tyrosine phosphorylation of an ITAM-like sequence by the activated Src family of kinases occurred, which resulted in the expression of docking sites for the Syk protein. Duan et al (28) revealed that Candida parapsilosis could stimulate the inflammatory response, increase the expression of Dectin-1, and activate NF- $\mathrm{NB}$ and MAPK signaling pathways in macrophages. Gringhuis et al (29) reported that Dectin-1 expressed on human DCs activated the Syk-dependent canonical NF- $\kappa \mathrm{B}$ subunits p65 and c-Rel. The results of the present study are consistent with these findings in which Dectin-1 in THP-1 macrophages recognized pathogens and induced the activation of Syk, in turn triggering the downstream molecules, $\mathrm{I} \kappa \mathrm{B} \alpha$ and $\mathrm{NF}-\kappa \mathrm{B}$. In the present study, it was observed that the phosphorylation levels of Syk, p65 and I $\mathrm{B} \alpha$ protein were increased, and p65 nuclear translocation was induced following stimulation of THP-1 macrophages with T. marneffei. These results suggested that T. marneffei could induce Syk-mediated activation of NF- $\kappa \mathrm{B}$ in THP-1 macrophages. In addition, it was revealed that silencing of Dectin-1 inhibited the phosphorylation of Syk, p65 and IкB $\alpha$ in THP-1 macrophages induced by T. marneffei spores. It was hypothesized that Dectin-1 may participate in the immunological defense against T. marneffei, and the Dectin-1/Syk/NF- $\mathrm{B}$ signaling pathway may serve an indispensable role in $T$. marneffei infection.

Inflammatory cytokines play a critical role in the development of fungal infectious diseases. Monocytes/macrophages are an important part of innate immunity against fungi, and they primarily produce cytokines, such as TNF- $\alpha$, IL-1, IL-6, IL-8 and granulocyte-colony stimulating factor $(30,31)$. TNF- $\alpha$ is a pro-inflammatory cytokine produced by immune cells, primarily $\mathrm{T}$ lymphocytes. TNF- $\alpha$ belongs to a family of both soluble and cell-bound cytokines that have a wide 
A

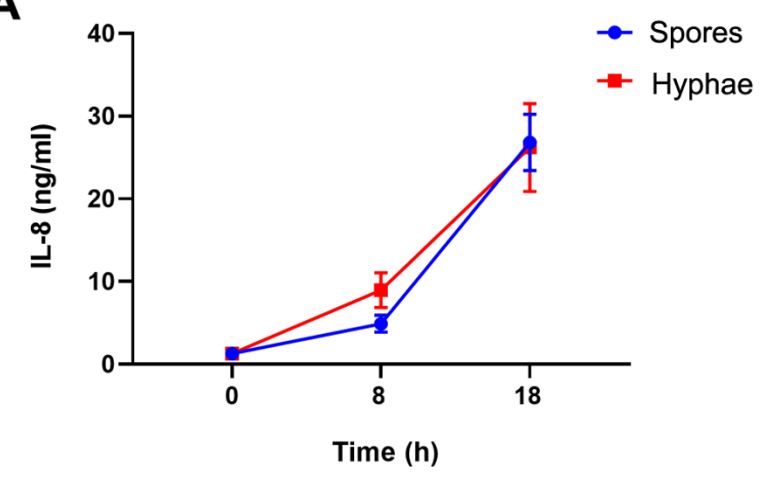

C

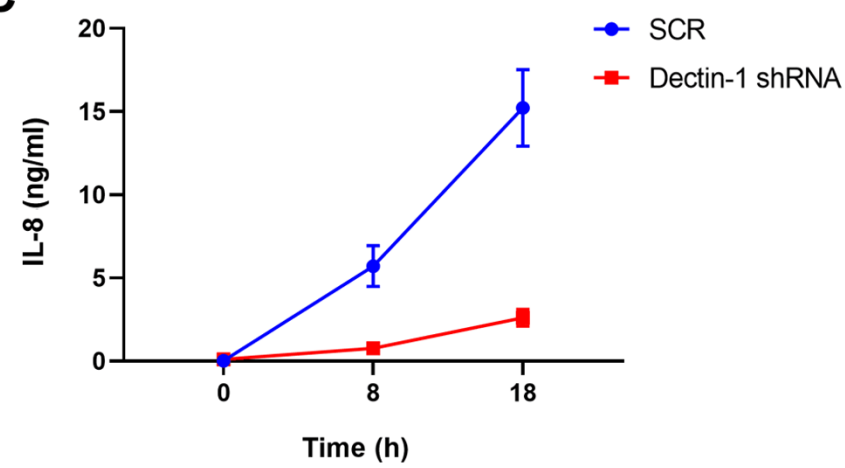

B

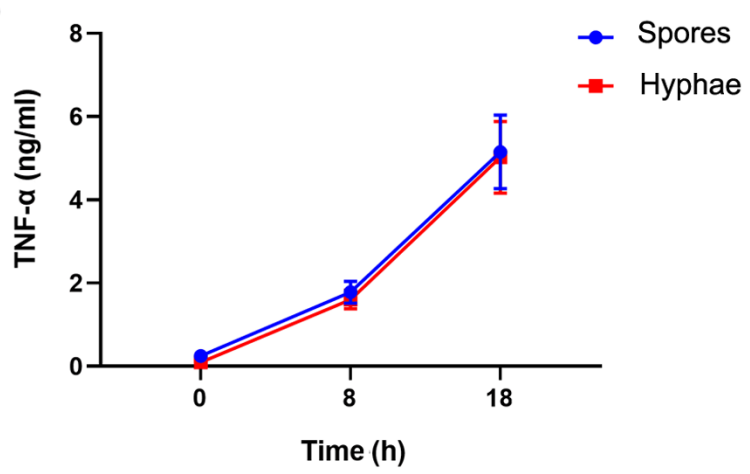

D

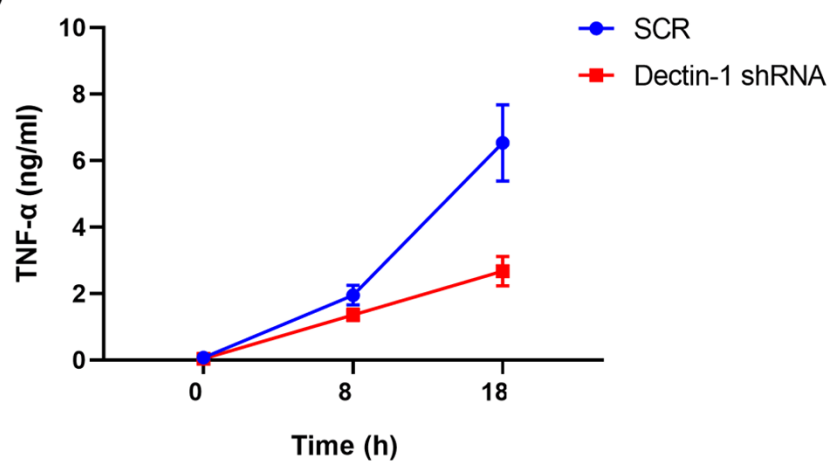

Figure 4. Knockdown of Dectin-1 inhibits cytokine release from macrophages. (A and B) THP-1 macrophages were exposed to Talaromyces marneffei spores or hyphae for 8 or $18 \mathrm{~h}$. The secretion of (A) IL- 8 and (B) TNF- $\alpha$ protein in the cell free culture supernatants was determined using ELISA. TNF- $\alpha$ and IL-8 were upregulated in THP-1 macrophages following stimulation with T. marneffei. (C and D) THP-1 macrophages were transfected with Dectin-1 shRNA or SCR shRNA control. Transfected cells were then incubated with T. marneffei spores for 8 or $18 \mathrm{~h}$. Levels of (C) IL-8 and (D) TNF- $\alpha$ secreted by the transfected cells were quantified using ELISA. Treatment with Dectin-1 shRNA before T. marneffei infection significantly reduced TNF- $\alpha$ and IL- 8 levels. Data are presented as the mean \pm standard deviation of three repeats. SCR, scrambled; shRNA, short hairpin RNA; Dectin-1, dendritic cell-associated C-type lectin-1.

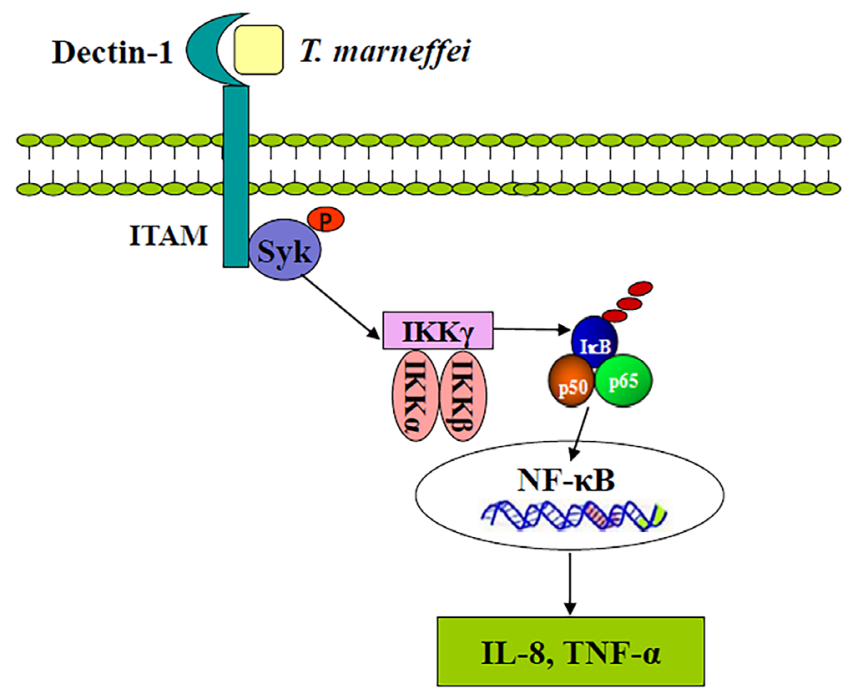

Figure 5. Schematic representation of Dectin-1 and its roles in the immune response to Talaromyces marneffei infection addressed in the present study. T. marneffei binds and activates Dectin-1 receptor, leading to activation of the NF- $\mathrm{KB}$ signaling pathway, including activation of IKK complexes, release of $\mathrm{p} 50 / \mathrm{p} 65$ complexes from the inhibitor complex with IкB $\alpha$, and translocation of the phosphorylated $\mathrm{p} 50 / \mathrm{p} 65$ heterocomplex to the nucleus, where the transcription of proinflammatory genes, such as the cytokines TNF- $\alpha$ and IL-8, is induced. Syk, spleen tyrosine kinase; Dectin-1, dendritic cell-associated C-type lectin-1; I $\kappa \mathrm{B} \alpha$, nuclear factor of $\kappa$ light polypeptide gene enhancer in B-cells inhibitor, $\alpha$; $p$, phosphorylated. range of functions, such as host defense, inflammation and apoptosis $(32,33)$. IL-8, also known as CXCL8, is a proinflammatory CXC chemokine involved in inflammatory reactions. The biological effects of IL-8 are mediated by two highly related chemokine receptors, CXCR1 (IL-8RA) and CXCR2 (IL-8RB). IL-8 exerts its function alongside other cytokines and chemokines, thus causing chemoattraction of leukocytes to sites of inflammation, recruitment and activation of neutrophils to phagocytosis, and bacterial clearance $(34,35)$. Li et al (36) demonstrated that Candida albicans could induce NF- $\kappa B$ activation and cytokine (TNF- $\alpha$ and IL-8) production. Furthermore, Hohl et al (37) identified that antibody-mediated blockade of Dectin-1 partially inhibited TNF- $\alpha /$ macrophage inflammatory protein- 2 induction by metabolically active conidia of Aspergillus fumigatus. The present study revealed that T. marneffe $i$ treatment in THP-1 macrophages resulted in increased levels of two pro-inflammatory cytokines (TNF- $\alpha$ and IL-8). Furthermore, Dectin-1 silencing in THP-1 macrophages with $T$. marneffei stimulation resulted in significantly decreased TNF- $\alpha$ and IL- 8 cytokine production. These results indicated that Dectin-1 is important in inducing the secretion of proinflammatory cytokines. Consistently, knockdown of Dectin-1 reduced inflammation by decreasing the levels of pro-inflammatory cytokines. Taken together, the results demonstrated the essential pro-inflammatory role of Dectin-1 in talaromycosis. 
In conclusion, the present study confirmed that Dectin-1 was involved in the recognition of $T$. marneffei on human macrophages, and contributed to their immunomodulatory capacity (Fig. 5). It was verified that T. marneffei induced immune responses by activation of Dectin-1 as well as the $\mathrm{NF}-\kappa \mathrm{B}$ signaling pathway in THP-1 macrophages. Dectin-1 was shown to be an important receptor for T. marneffei on THP-1 macrophages and it was revealed to be involved in the induction of a pro-inflammatory cytokine response. Therefore, it was hypothesized that regulating the expression of Dectin-1 receptor could interfere with the innate immune state of the host, and thus regulate the defense ability of the host against T. marneffei infection. Although the underlying molecular mechanisms remain to be elucidated, the present findings may partly explain the immune response associated with T. marneffei infection, and contributed to an improved understanding of the immune response against T. marneffei. By studying the precise responses to T. marneffei infection in macrophages, these findings may enable the further exploration and development of novel antifungal strategies. However, there are limitations of the present study. Dectin-1 expression was only investigated at the cellular level, and the mechanisms underlying the effects of Dectin-1 on T. marneffei infection were not evaluated in vivo. Therefore, the effects of Dectin-1 on T. marneffei infection must be further verified using in vivo experiments.

\section{Acknowledgements}

Not applicable.

\section{Funding}

The present study was supported by the National Natural Science Foundation of China (grant no. 81572052), the Natural Science Foundation of Jiangsu province, China (grant no. BK20151178), the Natural Science Foundation for Yang Scholars of Jiangsu province (grant no. BK20180184), the Health and Family Planning Commission for Yang Technology talents of Changzhou (grant no. QN201710) and the Young Talent Development Plan of Changzhou Health Commission (grant no. 2020-233).

\section{Availability of data and materials}

The datasets used and/or analyzed during the current study are available from the corresponding author on reasonable request.

\section{Authors' contributions}

YP, YC and WS performed the experiments. YP and YC prepared the manuscript. YaW, JM, WZ, HZ and WS analyzed the data. HZ, YuW, WZ and WS were responsible for study conception and design of the study. JM and YaW contributed to literature searching and processing. WZ and WS confirm the authenticity of all the raw data. All authors have read and approved the final manuscript.

\section{Ethics approval and consent to participate}

Not applicable.

\section{Patient consent for publication}

Not applicable.

\section{Competing interests}

The authors declare that they have no competing interests.

\section{References}

1. Capponi M, Segretain G and Sureau P: Penicillosis from Rhizomys sinensis. Bull Soc Pathol Exot Filiales 49: 418-421, 1956 (In French).

2. Segretain G: Penicillium marneffei n.sp., agent of a mycosis of the reticuloendothelial system. Mycopathol Mycol Appl 11: 327-353, 1959 (In French).

3. Boyce KJ and Andrianopoulos A: Fungal dimorphism: The switch from hyphae to yeast is a specialized morphogenetic adaptation allowing colonization of a host. FEMS Microbiol Rev 39: 797-811, 2015.

4. Cogliati M, Roverselli A, Boelaert JR, Taramelli D, Lombardi L and Viviani MA: Development of an in vitro macrophage system to assess Penicillium marneffei growth and susceptibility to nitric oxide. Infect Immun 65: 279-284, 1997.

5. Tsang CC, Lau SKP and Woo PCY: Sixty Years from Segretain's Description: What Have We Learned and Should Learn About the Basic Mycology of Talaromyces marneffei? Mycopathologia 184: 721-729, 2019.

6. Hu Y, Zhang J, Li X, Yang Y, Zhang Y, Ma J and Xi L: Penicillium marneffei infection: An emerging disease in mainland China. Mycopathologia 175: 57-67, 2013.

7. Le T, Huu Chi N, Kim Cuc NT, Manh Sieu TP, Shikuma CM, Farrar J and Day JN: AIDS-associated Penicillium marneffei infection of the central nervous system. Clin Infect Dis 51: 1458-1462, 2010.

8. Sellge G and Kufer TA: PRR-signaling pathways: Learning from microbial tactics. Semin Immunol 27: 75-84, 2015.

9. Akira S, Uematsu S and Takeuchi O: Pathogen recognition and innate immunity. Cell 124: 783-801, 2006.

10. Salazar F and Brown GD: Antifungal Innate Immunity: A Perspective from the Last 10 Years. J Innate Immun 10: 373-397, 2018.

11. Goyal S, Castrillón-Betancur JC, Klaile E and Slevogt H: The Interaction of Human Pathogenic Fungi With C-Type Lectin Receptors. Front Immunol 9: 1261, 2018.

12. Sancho D and Reis e Sousa C: Signaling by myeloid C-type lectin receptors in immunity and homeostasis. Annu Rev Immunol 30: 491-529, 2012.

13. Saijo S and Iwakura Y: Dectin-1 and Dectin-2 in innate immunity against fungi. Int Immunol 23: 467-472, 2011.

14. Wevers BA, Kaptein TM, Zijlstra-Willems EM, Theelen B, Boekhout T, Geijtenbeek TB and Gringhuis SI:Fungal engagement of the C-type lectin mincle suppresses dectin-1-induced antifungal immunity. Cell Host Microbe 15: 494-505, 2014.

15. Brown GD: Dectin-1: A signalling non-TLR pattern-recognition receptor. Nat Rev Immunol 6: 33-43, 2006.

16. Saijo S, Ikeda S, Yamabe K, Kakuta S, Ishigame H, Akitsu A, Fujikado N, Kusaka T, Kubo S, Chung SH, et al: Dectin-2 recognition of alpha-mannans and induction of Th17 cell differentiation is essential for host defense against Candida albicans. Immunity 32: 681-691, 2010.

17. Livak KJ and Schmittgen TD: Analysis of relative gene expression data using real-time quantitative PCR and the 2(-Delta Delta C(T)) Method. Methods 25: 402-408, 2001.

18. Limper AH, Adenis A, Le T and Harrison TS: Fungal infections in HIV/AIDS. Lancet Infect Dis 17: e334-e343, 2017.

19. Nakamura K, Miyazato A, Koguchi Y, Adachi Y, Ohno N, Saijo S, Iwakura Y, Takeda K, Akira S and Fujita J: Toll-like receptor 2 (TLR2) and dectin-1 contribute to the production of IL-12p40 by bone marrow-derived dendritic cells infected with Penicillium marneffei. Microbes Infect 10: 1223-1227, 2008.

20. Gantner BN, Simmons RM, Canavera SJ, Akira S and Underhill DM: Collaborative induction of inflammatory responses by dectin-1 and Toll-like receptor 2. J Exp Med 197: 1107-1117, 2003. 
21. Sun WK, Lu X, Li X, Sun QY, Su X, Song Y, Sun HM and Shi Y: Dectin-1 is inducible and plays a crucial role in Aspergillus-induced innate immune responses in human bronchial epithelial cells. Eur J Clin Microbiol Infect Dis 31: 2755-2764, 2012.

22. Cohen-Kedar S, Baram L, Elad H, Brazowski E, Guzner-Gur H and Dotan I: Human intestinal epithelial cells respond to $\beta$-glucans via Dectin-1 and Syk. Eur J Immunol 44: 3729-3740, 2014.

23. Hayden MS and Ghosh S: Shared principles in NF-kappaB signaling. Cell 132: 344-362, 2008.

24. Hinz $\mathrm{M}$ and Scheidereit $\mathrm{C}$ : The I $\kappa \mathrm{B}$ kinase complex in $\mathrm{NF}-\kappa \mathrm{B}$ regulation and beyond. EMBO Rep 15: 46-61, 2014.

25. Zhu LL, Zhao XQ, Jiang C, You Y, Chen XP, Jiang YY, Jia XM and Lin X: C-type lectin receptors Dectin-3 and Dectin-2 form a heterodimeric pattern-recognition receptor for host defense against fungal infection. Immunity 39: 324-334, 2013.

26. Sun H, Xu XY, Tian XL, Shao HT, Wu XD, Wang Q, Su X and Shi Y: Activation of $\mathrm{NF}-\kappa \mathrm{B}$ and respiratory burst following Aspergillus fumigatus stimulation of macrophages. Immunobiology 219: 25-36, 2014.

27. Rogers NC, Slack EC, Edwards AD, Nolte MA, Schulz O, Schweighoffer E, Williams DL, Gordon S, Tybulewicz VL, Brown GD, et al: Syk-dependent cytokine induction by Dectin-1 reveals a novel pattern recognition pathway for $\mathrm{C}$ type lectins. Immunity 22: 507-517, 2005.

28. Duan Z, Chen X, Du L, Liu C, Zeng R, Chen Q and Li M: Inflammation Induced by Candida parapsilosis in THP-1 Cells and Human Peripheral Blood Mononuclear Cells (PBMCs). Mycopathologia 182: 1015-1023, 2017.

29. Gringhuis SI, den Dunnen J, Litjens M, van der Vlist M, Wevers B Bruijns SC and Geijtenbeek TB: Dectin-1 directs T helper cell differentiation by controlling noncanonical NF-kappaB activation through Raf-1 and Syk. Nat Immunol 10: 203-213, 2009.

30. Koh TJ and DiPietro LA: Inflammation and wound healing: The role of the macrophage. Expert Rev Mol Med 13: e23, 2011.
31. Shapouri-Moghaddam A, Mohammadian S, Vazini $H$ Taghadosi M, Esmaeili SA, Mardani F, Seifi B, Mohammadi A, Afshari JT and Sahebkar A: Macrophage plasticity, polarization, and function in health and disease. J Cell Physiol 233: 6425-6440, 2018.

32. Idriss HT and Naismith JH: TNF alpha and the TNF receptor superfamily: Structure-function relationship(s). Microsc Res Tech 50: 184-195, 2000

33. Aggarwal BB, Gupta SC and Kim JH: Historical perspectives on tumor necrosis factor and its superfamily: 25 years later, a golden journey. Blood 119: 651-665, 2012.

34. Akdis M, Aab A, Altunbulakli C, Azkur K, Costa RA, Crameri R, Duan S, Eiwegger T, Eljaszewicz A, Ferstl R, et al: Interleukins (from IL-1 to IL-38), interferons, transforming growth factor $\beta$, and TNF- $\alpha$ : Receptors, functions, and roles in diseases. J Allergy Clin Immunol 138: 984-1010, 2016.

35. Zhang $\mathrm{W}$ and Chen H: The study on the interleukin-8 (IL-8)]. Sheng Wu Yi Xue Gong Cheng Xue Za Zhi 19: 697-702, 2002 (In Chinese).

36. Li M, Liu ZH, Chen Q, Zhou WQ, Yu MW, Lü GX, Lü XL, Shen YN, Liu WD and Wu SX: Insoluble beta-glucan from the cell wall of Candida albicans induces immune responses of human THP-1 monocytes through Dectin-1. Chin Med J (Engl) 122: 496-501, 2009.

37. Hohl TM, Van Epps HL, Rivera A, Morgan LA, Chen PL, Feldmesser M and Pamer EG: Aspergillus fumigatus triggers inflammatory responses by stage-specific beta-glucan display. PLoS Pathog 1: e30, 2005.

c) (i) $\Theta$ This work is licensed under a Creative Commons cc) Attribution-NonCommercial-NoDerivatives 4.0 International (CC BY-NC-ND 4.0) License. 